\title{
e-Blood
}

\section{Pathophysiology of sickle cell disease is mirrored by the red blood cell metabolome}

\author{
Dhouha Darghouth, ${ }^{1-4}$ Bérengère Koehl, ${ }^{1-4}$ Geoffrey Madalinski, ${ }^{5}$ Jean-François Heilier, ${ }^{5,6}$ Petra Bovee, ${ }^{7}$ Ying Xu, $^{3}$ \\ Marie-Françoise Olivier, ${ }^{1}$ Pablo Bartolucci, ${ }^{8,9}$ Malika Benkerrou, ${ }^{10}$ Serge Pissard, ${ }^{11}$ Yves Colin, ${ }^{8}$ Frederic Galacteros, ${ }^{9}$ \\ Giel Bosman, ${ }^{*}$ Christophe Junot, ${ }^{5}$ and ${ }^{*}$ Paul-Henri Roméo ${ }^{1-4}$ \\ ${ }^{1}$ Commissariat à l'Energie Atomique/Direction du Sciences et du vivant/Institut de radiobiologie cellulaire et moléculaire/Laboratoire de Recherche sur la

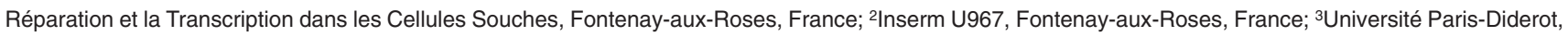 \\ Paris, France; ‘Université Paris-Sud, Paris, France; ${ }^{5}$ Commissariat à l'Energie Atomique/Direction du Sciences et du vivant Institut de biologie et de \\ technologies de Saclay/Service de Pharmacologie et d'Immunoanalyse, Saclay, Gif-sur-Yvette, France; 6 Université Catholique de Louvain, Louvain Center for \\ Toxicology and Applied Pharmacology, Brussels, Belgium; ${ }^{7}$ Department of Biochemistry, Radboud University Nijmegen Medical Centre and Nijmegen Centre for \\ Molecular Life Sciences, Nijmegen, The Netherlands; ${ }^{8}$ Inserm, UMRS 665, Paris, France; 9Unité des Maladies Génétiques du Globule Rouge, Hôpital \\ Henri-Mondor, Assistance Publique des Hôpitaux de Paris, Université Paris, Créteil, France; ${ }^{10}$ Assistance Publique des Hôpitaux de Paris, Service \\ d'Hématologie-Oncologie Pédiatrique, Hôpital Robert Debré, Paris, France; and ${ }^{11}$ Service de Génétique et de Biochimie, Centre Hospitalier Universitaire Hôpital \\ Henri Mondor, Université Paris, Créteil, France
}

\begin{abstract}
Emerging metabolomic tools can now be used to establish metabolic signatures of specialized circulating hematopoietic cells in physiologic or pathologic conditions and in human hematologic diseases. To determine metabolomes of normal and sickle cell erythrocytes, we used an extraction method of erythrocytes metabolites coupled with a liquid chromatography-mass spectrometrybased metabolite profiling method. Comparison of these 2 metabolomes identified major changes in metabolites produced
\end{abstract}

by (1) endogenous glycolysis characterized by accumulation of many glycolytic intermediates; (2) endogenous glutathione and ascorbate metabolisms characterized by accumulation of ascorbate metabolism intermediates, such as diketogulonic acid and decreased levels of both glutathione and glutathione disulfide; (3) membrane turnover, such as carnitine, or membrane transport characteristics, such as amino acids; and (4) exogenous arginine and NO metabolisms, such as spermine, spermidine, or citrulline. Finally, metabolomic analysis of young and old normal red blood cells indicates metabolites whose levels are directly related to sickle cell disease. These results show the relevance of metabolic profiling for the follow-up of sickle cell patients or other red blood cell diseases and pinpoint the importance of metabolomics to further depict the pathophysiology of human hematologic diseases. (Blood. 2011; 117(6):e57-e66)

\section{Introduction}

Metabolome is defined as the complete set of metabolites present in a given biologic system that can be unicellular organism, organ, tissue, cell, or biologically relevant liquid compartments. Complementary to genomics or proteomics, the aim of metabolome analysis is to describe qualitatively and quantitatively the final products of cellular regulatory pathways and can be seen as the ultimate response of a biologic system to genetic factors and/or environmental changes. ${ }^{1}$ Presently, metabolomics is used to describe metabolites present in simple unicellular organisms, such as Escherichia coli, ${ }^{2}$ or in important biologic fluids, such as plasma or urine, ${ }^{3,4}$ but the cross-talks between cells in tissues or the complex metabolism in mammalian cells make the interpretation of metabolomics data challenging in human, although important metabolites involved in pathogenesis of cancer, ${ }^{5,6}$ diabetes, ${ }^{7}$ and cardiovascu$1 \mathrm{ar}^{8,9}$ and mitochondrial disease $\mathrm{s}^{10}$ are described.

Mammalian mature red blood cell (RBC) is a cell without nucleus or cytoplasmic organelles, such as mitochondria or ribosomes, easy to collect and to purify in large quantity. In human, during their 120-day life span, RBCs are perfectly adapted to oxygen, carbon dioxide, and proton transport. RBCs have also an important role in interorgan transport of metabolites, such as amino acid transport to muscles resulting from numerous amino acids receptors on $\mathrm{RBC}$ membranes. $\mathrm{RBCs}$ are also used as reporters of exogenous metabolisms as exemplified by the level of hemoglobin A1c, which is a measure of erythrocyte hemoglobin glycation and reflects mean glycemia for the previous 3 months in humans. RBC metabolism is supposed to be restricted to glycolysis, nucleotide catabolism, glutathione metabolism, ${ }^{11}$ and protection of hemoglobin, enzymes and proteins that protect $\mathrm{RBC}$ membrane against oxidation, ${ }^{12}$ but a recent exploration of the human $\mathrm{RBC}$ proteome has identified unexpected minor proteins that are involved in other metabolic pathways that need to be characterized. ${ }^{13}$ Thus, RBCs in whole blood could be one of the most suitable natural systems to investigate dynamic modification of metabolome in human physiology or in human diseases.

Sickle cell anemia, one of the most common autosomal recessive diseases in the world, is caused by a single nucleotide substitution (GTG $>\mathrm{G} A \mathrm{G})$ at the sixth codon of the human $\beta$-globin gene. This point mutation results in well known hemolytic and vaso-occlusive complications that characterize sickle cell
Submitted July 30, 2010; accepted November 9, 2010. Prepublished online as Blood First Edition paper, December 6, 2010; DOI 10.1182/blood-201007-299636.

${ }^{\star}$ P.-H.R. and C.J. contributed equally to this study.
The online version of this article contains a data supplement.

The publication costs of this article were defrayed in part by page charge payment. Therefore, and solely to indicate this fact, this article is hereby marked "advertisement" in accordance with 18 USC section 1734.

(C) 2011 by The American Society of Hematology 
Table 1. Summary of clinical data for $\mathrm{Hb}-\mathrm{SS}$ subjects

\begin{tabular}{|c|c|c|c|c|c|c|c|c|}
\hline Subject no. & Sex & Age, y & $\mathrm{Hb}, \mathrm{g} / \mathrm{dL}$ & $\mathrm{Hb} F, \%$ & MCV, fL & Reticulocytes, $\times 10^{9} / \mathrm{L}(\%)$ & WBCs, $\times 10^{9} / \mathrm{L}$ & Platelets, $\times 10^{9} / \mathrm{L}$ \\
\hline SCD 1 & $\mathrm{~F}$ & 25 & 9.4 & 5.3 & 85 & $235(6.9)$ & 9.6 & 356 \\
\hline SCD 2 & M & 34 & 8.6 & $N D^{d}$ & 92 & $351(12.5)$ & 10.1 & 265 \\
\hline SCD 3 & $\mathrm{~F}$ & 23 & 7.9 & ND & 86 & $261(9.6)$ & 7.8 & 377 \\
\hline SCD 4 & $\mathrm{~F}$ & 28 & 9.4 & 6.2 & 93 & $351(12.5)$ & 11.2 & 380 \\
\hline SCD 5 & M & 20 & 11.4 & 10.4 & 89 & $250(6.8)$ & 11.1 & 505 \\
\hline SCD 6 & $M$ & 29 & 8.1 & ND & 90 & ND & 11.2 & 504 \\
\hline SCD 7 & $\mathrm{~F}$ & 24 & 10.2 & 9.9 & 88 & $214(6.1)$ & 9.7 & 499 \\
\hline SCD 8 & $M$ & 38 & 11.1 & 15.9 & 109 & ND & 12.4 & 275 \\
\hline SCD 9 & $\mathrm{~F}$ & 55 & 8.1 & ND & 91 & ND & 7.9 & 164 \\
\hline SCD 10 & $M$ & 26 & 9.7 & 1.6 & 70 & $205(4.7)$ & 13.5 & 496 \\
\hline SCD 11 & $\mathrm{~F}$ & 40 & 8.7 & ND & 100 & $174(6.7)$ & 9.3 & 349 \\
\hline SCD 12 & $M$ & 28 & 9 & ND & 85 & $229(7.4)$ & 12.2 & 389 \\
\hline SCD 13 & $\mathrm{~F}$ & 19 & 9.1 & 12.7 & 99 & $296(11)$ & 14.4 & 628 \\
\hline SCD 14 & $M$ & 46 & 7.3 & 5.9 & 90 & $323(14)$ & 9.3 & 288 \\
\hline SCD 15 & $M$ & 27 & 8.2 & 0.9 & 75 & $139(4.2)$ & 6.4 & 256 \\
\hline SCD 16 & M & 38 & 6.1 & ND & 93 & $218(11.4)$ & 12.8 & 292 \\
\hline SCD 17 & $M$ & 34 & 8.6 & ND & 92 & $351(12.5)$ & 10.1 & 265 \\
\hline SCD 18 & $M$ & 45 & 10.5 & ND & 84 & $216(5.7)$ & 8.9 & 263 \\
\hline SCD 19 & $\mathrm{~F}$ & 46 & 9.3 & 11.2 & 89 & $231(7.2)$ & 12.4 & 379 \\
\hline SCD 20 & $M$ & 39 & 7.7 & ND & 81 & $448(16)$ & 11.3 & 259 \\
\hline SCD 21 & $M$ & 33 & 11.2 & 9 & 83 & $228(5.4)$ & 9.3 & 262 \\
\hline SCD 22 & $\mathrm{~F}$ & 28 & 9.5 & 8.8 & 85 & $336(9.9)$ & 12.9 & 389 \\
\hline SCD 23 & $M$ & 31 & 11.3 & ND & 83 & $184(4.4)$ & 7.8 & 317 \\
\hline SCD 24 & $M$ & 33 & 8.9 & 6.7 & 87 & $163(5.2)$ & 8.0 & 299 \\
\hline SCD 25 & $\mathrm{~F}$ & 38 & 7.6 & 2.9 & 90 & $224(9.3)$ & 11.6 & 359 \\
\hline SCD 26 & $\mathrm{~F}$ & 42 & 9.6 & ND & 110 & $210(8.2)$ & 10.1 & 445 \\
\hline SCD 27 & $M$ & 46 & 10.5 & 1.7 & 80 & $350(8.5)$ & 7.8 & 275 \\
\hline SCD 28 & $\mathrm{~F}$ & 44 & 8.2 & ND & 108 & 249 (11.3) & 13.9 & 422 \\
\hline
\end{tabular}

F indicates female; M, male; Hb, hemoglobin; MCV, mean corpuscular volume; WBCs, white blood cells; and ND, not determined.

disease (SCD). Although the polymerization of sickle hemoglobin $(\mathrm{HbS})$ is the primary event in the pathogenesis of SCD, the pathophysiology of SCD is far more complex and involves endogenous and exogenous dysfunctions. ${ }^{14} \mathrm{HbS}$ polymerization results in cellular alterations of the RBCs, such as membrane alterations that shorten the red cell life span and lead to vascular occlusion with induced damage in the infracted organ. In addition, adherence of the RBCs and other cellular elements to vascular endothelium, proinflammatory events and scavenging of nitric oxide are involved in the pathogenesis of several SCD-related complications. ${ }^{15}$ Until recently, SCD treatments have remained limited, but the availability of several novel therapeutic agents in various phases of development is encouraging. ${ }^{16}$ The complex pathophysiology of SCD makes unlikely that a single therapeutic agent will prevent or reverse all SCD complications and metabolomic analysis might help in the characterization of the endogenous and exogenous effects of the new treatments.

There are very few reports providing comprehensive measurements of metabolites present in the $\mathrm{RBCs}^{17}$ and no reports on global metabolites changes associated with SCD. The aim of this study was to develop a simple and comprehensive strategy for metabolite extraction and metabolome analysis of purified human RBCs from normal person and sickle cell-affected patients and also to determine pathways that might be of interest to prevent vaso-occlusion and to monitor the effects of new drugs on SCD.

\section{Methods}

\section{Biologic samples}

For this study, blood samples were collected from a cohort of 28 adult SCD patients with hemoglobin SS disease (Table 1) and 24 healthy adults (controls). Written informed consent was obtained from all patients and controls in accordance with the Declaration of Helsinki. All protocols were approved by Inserm.

The SCD patients were enrolled in the Sickle Cell Disease Center in Henri Mondor Hospital. Excluded patients were those with hemoglobin SC disease or $\mathrm{S}^{+}$thalassemia, patients who received hydroxyurea treatment and patients transfused with RBCs in the last 3 months. Patients who were clinically diagnosed at the time of medical consultation with bacterial infection or vaso-occlusive symptoms were also excluded. The SCD patient group contains 12 women and 16 men $($ sex ratio $=1.33)$, and the average age is $34.3 \pm 9.1$ years. For the control group, the average age is $33.3 \pm 8.9$ years and the sex ratio is 1 .

Blood samples were collected into tubes containing ethylene diamine tetraacetic acid and kept at $4^{\circ} \mathrm{C}$ until metabolite extraction in the hours after blood sampling.

\section{Preparation of RBC lysates and metabolite extraction}

Whole blood was centrifuged at $120 \mathrm{~g}$ for 15 minutes at room temperature, and the supernatant containing platelets was discarded. To isolate the RBCs from leukocytes, a cellulose column was used as follows: $400 \mathrm{mg}$ of $\alpha$-cellulose (Sigma-Aldrich) and $400 \mathrm{mg}$ of Sigmacell Cellulose, Type 50 (Sigma-Aldrich) were mixed in an isotonic sodium chloride $(0.9 \% \mathrm{NaCl})$ solution. The barrel was removed from a $5-\mathrm{mL}$ syringe and fixed in a vertical position with the outlet pointing downward. A small piece of glass wool was placed at the bottom of the syringe, and the well-mixed cellulose was poured until the 5-mL mark; $1 \mathrm{~mL}$ of pelleted RBCs mixed with $1 \mathrm{~mL}$ of sodium chloride solution were gently poured on top of the column. The RBCs were then drained through the column using 5 to $10 \mathrm{~mL}$ of a $0.9 \%$ $\mathrm{NaCl}$ solution. The effluent was collected into one 15-mL Falcon tube. After this chromatography, the RBC purity was higher than $99.99 \%$. RBC suspensions obtained were centrifuged at $1430 \mathrm{~g}$ for 3 minutes at $4^{\circ} \mathrm{C}$, and the supernatant was discarded. A total of $4 \times 10^{9}$ purified RBCs were transferred, suspended in Milli-Q water in twice of the initial volume of packed cells, and boiled for 3 minutes. After centrifugation at $1430 \mathrm{~g}$ for 3 minutes at $4^{\circ} \mathrm{C}$, the supernatant was ultrafiltered with centrifugal filter 
devices, molecular weight cutoff of 30000 followed by 10000 (Amicon Ultra-4 centrifugal filter devices) at $4000 \mathrm{~g}$ for 20 minutes at $20^{\circ} \mathrm{C} .{ }^{18}$ The final ultrafiltrate was frozen at $-80^{\circ} \mathrm{C}$ until mass spectrometry analysis. Before injection into the chromatographic system, the samples were diluted 1:1 with Milli-Q water. A quality control sample was obtained by pooling RBC samples with a mixture of 8 internal standards (supplemental Table 1, available on the Blood Web site; see the Supplemental Materials link at the top of the online article).

\section{Liquid chromatography coupled to electrospray-LTQ-Orbitrap mass spectrometry}

A total of $10 \mu \mathrm{L}$ of samples was injected and separated on a $2.1 \times 150-\mathrm{mm}$ Hypersil gold C18 1.9- $\mu \mathrm{m}$ column (Thermo Electron) equipped with an on-line prefilter (Interchim). The flow rate was $0.5 \mathrm{~mL} / \mathrm{min}$ with mobile phases A (100\% water) and B (100\% acetonitrile), both containing $0.1 \%$ formic acid. The gradient consisted of an isocratic step of 2 minutes at $100 \%$ phase A, followed by a linear gradient from $0 \%$ to $100 \%$ of phase B for the next 13 minutes, before returning to $100 \%$ A for 4 minutes. Mass spectrometric detection was performed using an LTQ-Orbitrap hybrid mass spectrometer (Thermo Electron) fitted with an electrospray source operated in the positive and negative ion modes. The detection was achieved from 50 to $1000 \mathrm{u}$ at the maximum resolving power of 30000 (expressed as full width at half maximum for an ion at $400 \mathrm{U}$ ). The mass spectrometer was operated with capillary voltage at $4 \mathrm{kV}$ and capillary temperature at $275^{\circ} \mathrm{C}$. The sheath gas pressure and the auxiliary gas pressure were set, respectively, at 45 and 10 arbitrary units with nitrogen gas. Samples were randomly analyzed together by ultra-high performance liquid chromatography coupled to mass spectrometry (UHPLC/MS), and a quality control sample was injected every $10 \mathrm{RBC}$ samples to check the performance of the analytical system in terms of retention times, accurate mass measurements, and signal intensities.

Collision-induced dissociation spectra were acquired using the datadependent scanning function for identification purpose. The scan event cycle composed a full scan mass spectrum at a resolution power of 30000 and one or 2 data-dependent $\left(\mathrm{MS}^{2}\right.$ and $\mathrm{MS}^{3}$ ) events acquired with a resolution set to 7500 . Microscan count was set to unity, and a repeat count for dynamic exclusion was set to $3 . \mathrm{MS}^{\mathrm{n}}$ acquisition parameters were an isolation width of $1 \mathrm{u}$, normalized collision energy ranging from $15 \%$ to $45 \%$, and an activation time of $30 \mathrm{~ms}$

\section{Data processing and multivariate statistical analysis}

Our data processing procedure included 2 main steps: (1) Automatic peak detection. Raw data were analyzed using the XCMS software Version 1.14.1 running under $\mathrm{R}$ Version 2.8.1, ${ }^{19}$ (http://www.bioconductor.org/ packages/bioc/html/xcms.html). The UHPLC/MS data were peak-detected and noise-reduced in both the LC and MS domains to exclude peaks related to noise. The resulting datasets consisted of a single matrix containing features with $\mathrm{m} / \mathrm{z}$-retention time pair as an identifier and their intensity values for each sample. (2) Annotation of the datasets. The datasets resulting from the XCMS process were then annotated using tools developed in-house.

The resulting data from the XCMS process were first mean-centered and scaled either to unit variance or to Pareto variance, then introduced into SIMCA-P11 (Umetrics) for multivariate analyses by unsupervised principal component analysis (PCA) or by supervised projection to latent structurediscriminant analysis (PLS-DA). The PLS-DA models were validated using the cross-validation function of SIMCA-P11 and by permutation tests $(\mathrm{k}=100)$. In the 2 analyses, the first principal component $\mathrm{t}[1]$ accounts for as much of the variability in the data as possible, and the second $t[2]$ and third $\mathrm{t}[3]$ components account for as much of the remaining variability. ${ }^{20}$

\section{Results}

\section{A simple method to investigate the RBC metabolome}

We used a simple RBC metabolite extraction method previously described ${ }^{18}$ and UHPLC coupled to a LTQ-Orbitrap discovery mass spectrometer (Thermo Electron) fitted with an electrospray source to investigate human RBC metabolome. Detection of metabolites was achieved by picking their accurate masses from complex and information-rich metabolite fingerprints using an automatic peak detection software. Metabolites of biologic relevance were then highlighted using multivariate statistical analyses and identified with their elemental composition provided by accurate mass measurements and with complementary $\mathrm{MS}^{\mathrm{n}}$ experiments. The analytical method was validated using 3 criteria: intra-assay precision, impact of freeze-thaw cycles of $\mathrm{RBC}$ extracts on metabolite detection, and linearity of signal intensities as a function of sample dilution.

The intra-assay precision study determines whether the analytes are measured with an acceptable variability within one experiment. It was achieved by injecting an RBC extract spiked with a mixture of 8 xenobiotics used as internal reference every 10 samples to assess performances of the chromatographic column and the mass spectrometer during the experiments. Chromatographic retention times were stable in the course of experiments, with coefficients of variation less than $2 \%$ (supplemental Table 1). As for signal areas, coefficients of variation were less than $25 \%$; for all compounds except for one for which the coefficient of variation was $36 \%$ (values obtained with metformin, supplemental Table 1). Furthermore, each compound exhibited area values within 2 SD range, indicating that the MS signal was stable within experiments (supplemental Figure 1A-B).

The impact of freeze-thaw cycles of RBC extract on metabolite levels was determined with samples from 5 healthy volunteer donors. These extracts were subjected to 3 consecutive freeze-thaw cycles before UHPLC/MS analysis. The chromatograms were processed by automatic peak detection software and then submitted to PCA, an unsupervised projection method used to visualize the structure of the dataset. The scores plot indicated that there is no effect of freeze-thaw cycles and that interindividual variability is the main source of variance in the samples (Figure 1A). This absence of effects of freeze-thaw cycles on RBC metabolome was also confirmed on selected metabolites (Figure 1B).

Although automatic peak detection software is expected to report as many analytically relevant features as possible, artifactual signals (ie, noise signals that are detected as real signals) are generated during the detection process $(\sim 400$ for 100 relevant signals). ${ }^{21}$ These artifactual signals can be highlighted and removed as their intensities do not correlate with the dilution factor. ${ }^{21,22}$ The linearity of the signal response was investigated to search for such signals and to ensure that the variations in ion abundances observed in the course of metabolomic studies can be detected despite the occurrence of ion suppression effects. ${ }^{23}$ Among 3000 signals that could be detected in the positive and negative ion modes, more than 1700, including all the identified metabolites, exhibited coefficient correlation more than 0.7 and were thus found to be analytically relevant (Figure 1C).

\section{RBC metabolome revisited by UHPLC/MS}

The processing of a dataset of 52 UHPLC/MS chromatograms of $\mathrm{RBC}$ extracts by automatic detection software led to the detection of few thousand features in both positive and negative ion mode. A few hundred of them were considered as analytically relevant and were thus further considered. Data annotation procedures ("Data processing and multivariate statistical analysis") led to the characterization of 89 metabolites: 46 metabolites were identified on the basis of the similarity of their retention time, accurate mass, and also $\mathrm{MS}^{\mathrm{n}}$ spectra to those of authentic standards, and the other 
A

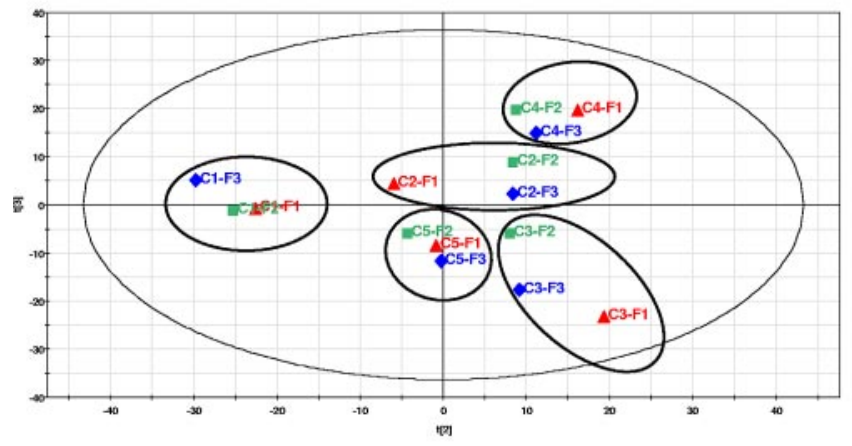

B
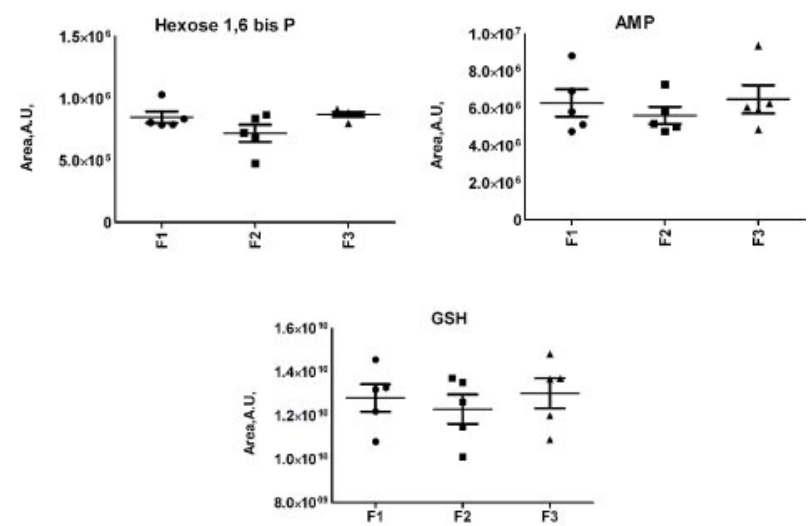

C

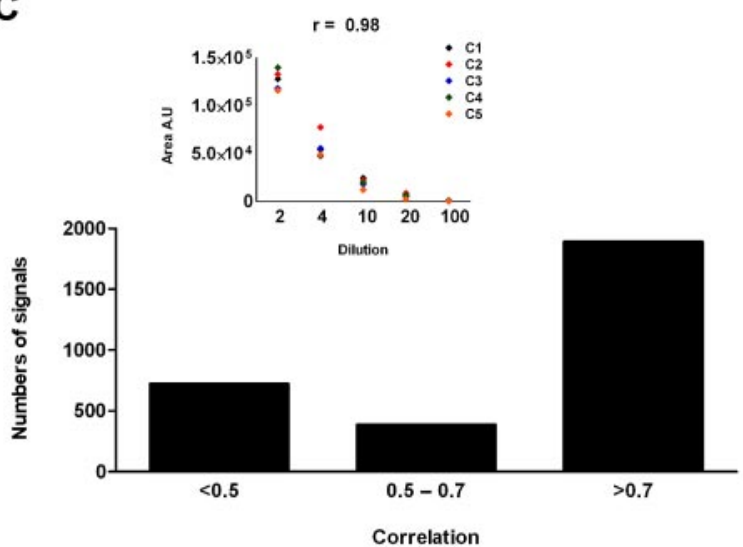

Figure 1. Validation of the LC/MS method. (A) Principal analysis score plots (PCA) for RBC extracts obtained from 5 healthy individual donors who were subjected to 3 consecutive freeze-thaw cycles before LC/MS analysis. The chromatograms were processed by automatic peak detection software. The variables (ie, couples of $\mathrm{m} / \mathrm{z}$ ratio, retention time) contained in the resulting data matrix were mean-centered and scaled to unit variance before PCA. Data for each person are circled, and the samples corresponding to the first, second, and third freeze-thaw cycles are red, green, and blue, respectively. (B) Levels of the 3 indicated metabolites as a function of the number $(\mathrm{F} 1=1, \mathrm{~F} 2=2$, and $\mathrm{F} 3=3)$ of freeze-thaw cycles. A.U. indicates arbitrary units. (C) More than 1700 signals among 3000 detected in the positive and negative ionization mode exhibited coefficient correlations more than 0.7 with the dilution factor. (Inset) An example of correlation between UHPLC/MS areas and dilution of RBCs samples. C1 to C5 indicate control persons.

metabolites were putatively annotated according to the definition established by the Metabolomics Standard Initiative. ${ }^{24}$ No reference compound was available at the laboratory for these metabolites, but the matching was based on the elemental formula obtained from accurate mass measurements and, for some of them, on the basis of the interpretation of fragmentation spectra (supplemental Table 2).

The RBC metabolome was not only restricted to metabolites produced by metabolic pathways related to vital functions in RBCs, such as glycolysis, nucleotide, or glutathione metabolism, but also contained amino acids, polyamines, small peptides, vitamins, and carnitine derivatives (Figure 2). Some of these metabolites are produced by RBC enzymes, ${ }^{13}$ whereas others such as amino acids might come from external sources such as plasma or other cell types.

\section{RBC metabolome of sickle cell patients}

RBC extracts of 28 SCD patients and 24 age- and sex-matched controls were analyzed by LC/MS with detection in negative and positive ion modes. Processing of the metabolic fingerprints led to 1524 and 785 relevant signals in the negative and positive ion modes, respectively. The resulting data matrices were subjected to PCA. The PCA score plots discriminated metabolic fingerprints of healthy subjects from those of SCD patients on the first component for the data recorded in the negative ion mode, indicating that most of the variance of the dataset could be explained by the disease (Figure 3A), whereas the separation was less obvious for the data acquired in the positive ion mode because it only occurred on the second component (Figure 3B). These data indicate that SCD was the main source of variance in the dataset. Of note, 5 SCD samples did not cluster with the other SCD patients in PCA score plots corresponding to the positive mode of detection (Figure 3B). As we could not find any methodologically or clinically relevant explanation, these samples were then removed from the dataset. No difference between the metabolomes of men and women with SCD could be evidenced (data not shown). Finally, the discrimination between SCD patients and controls observed on the PCA score plots was not related to reticulocyte count because metabolic fingerprints from SCD patients with low reticulocyte count $(<4 \%)$ were not statistically different from that of SCD patients with high reticulocyte count ( $>11 \%$; data not shown).

Although fresh samples were used for this proof-of-concept study, many RBC samples of biologic relevance are stored frozen at $-196^{\circ} \mathrm{C}$ in glycerol..$^{25}$ To determine the effects of freezing RBCs on their metabolome, blood samples from SCD patients and controls were collected and divided in 2 aliquots, one being immediately extracted and the other frozen at $-196^{\circ} \mathrm{C}$ in glycerol before extraction. Similar clustering (Figure 3C) and concentration trends (Figure 3D) were obtained from the statistical analysis of the 


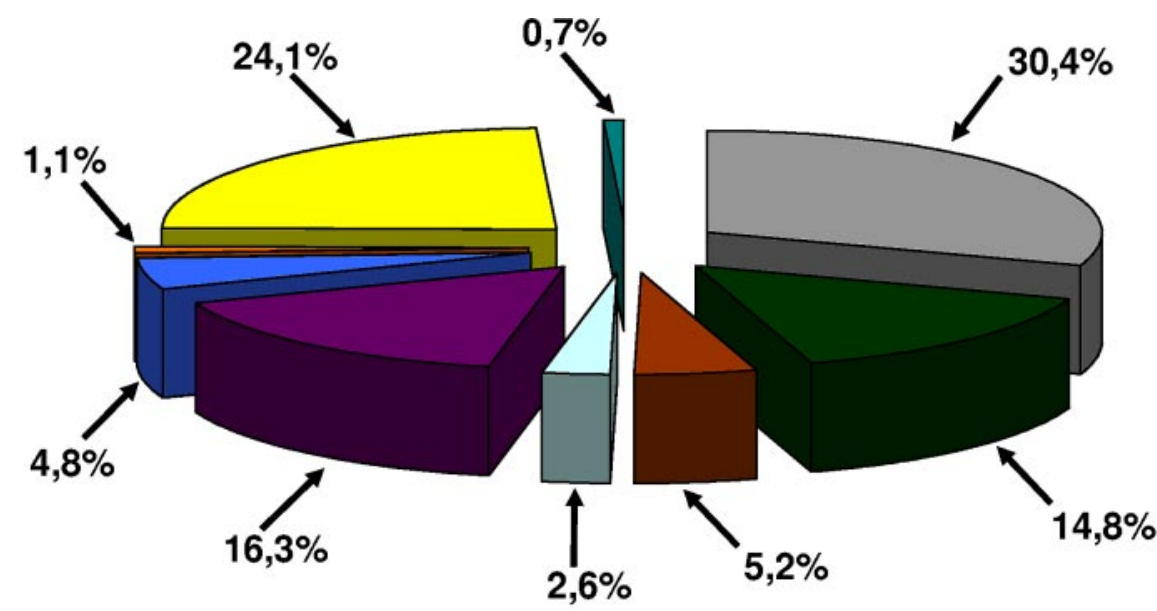

$\square$ Amino Acids
$\square$ Carboxylic Acids
$\square$ Carnitines and derivatives
$\square$ Nucleotides
$\square$ Others
$\square$ Polyamines
$\square$ Small peptides
$\square$ Sugar and derivatives
$\square$ Vitamins

Figure 2. The RBC metabolome revisited by LC/MS. The analysis of LC/MS data led to the characterization of 89 metabolites in RBC metabolome Shown are the percentages of the different metabolites that were identified in this study.

UHPLC/MS fingerprints, indicating that RBC storage at $-196^{\circ} \mathrm{C}$ in glycerol has a limited impact on the recorded metabolic data.

PLS-DA, a supervised method, was then used to improve the clustering observed with PCA and to facilitate the isolation of the variables responsible for the discrimination between SCD and control samples. Two-component PLS-DA models were built for each experimental condition (supplemental Figures 2A, 3A) for the detection in the negative and positive ion modes, respectively. The relevance of these models was evaluated by 2 parameters: (1) R2(Y), corresponding to the proportion of the variance of the response variable (ie, occurrence of the disease) that is explained by the model, and (2) Q2(cum), expressing the cumulative proportion of the variance of the variables that can be predicted by the model. The values of $\mathrm{R} 2(\mathrm{Y})$ were 0.9 and 0.8 , for negative and positive ion detection, respectively, whereas Q2(cum) values were more than 0.8 and 0.6 , for negative and positive ion detection, respectively, thus indicating that most of the variance related to our response variable is explained by the PLS-DA model. These models were also subjected to permutation tests to check for their validity and their degree of overfitting. Permutation of data from SCD patients related to data from healthy subjects induced dramatic decreases of the performance of the models, thus confirming their validity (supplemental Figures 2B, 3B).

Thirty-one metabolites exhibited significantly increased (Figure 4A; supplemental Tables 3-7) or decreased (Figure 4B; supplemental Tables 3-7) concentrations in RBCs from healthy subjects or from SCD patients ( $\mathrm{HbS}$ cells). Accumulations of intermediate products of the glycolytic and the pentose phosphate pathways, such as the hexose-phosphate (ie, fructose-6-phosphate, glucose-1phosphate, or glucose-6-phosphate), glyceraldehyde-3-phosphate or phosphoenolpyruvic acid were observed in $\mathrm{HbS}$ cells (supplemental Table 3), whereas concentrations of others, such as phosphoglycerate (ie, 2-or 3-phosphoglycerate), or pyruvate did not vary. The level of 2,3-diphosphoglycerate (2,3-DPG), which is produced by the Rappoport-Luebering shunt that is specific of RBCs, ${ }^{26}$ was also increased in $\mathrm{HbS}$ cells. Finally, the levels of malate, which can be synthesized from pyruvate (the end-product of glycolysis), were higher in HbS cells than in control cells, although we did not find any significant change in lactate concentration, the other metabolite that can be produced from pyruvate.

The main oxidative damages control system in RBCs, the glutathione pathway, was altered in $\mathrm{HbS}$ cells (supplemental Table 4). In HbS cells, reduced glutathione (GSH) and oxidized gluta- thione (GSSG) concentrations were significantly decreased, whereas the concentrations of their precursors (glutamine, glutamate, and glycine) were increased. The concentration of niacinamide, the substrate for nicotinamide adenine dinucleotide phosphate (NADP, an important cofactor of glutathione metabolism) synthesis, was decreased in HbS cells (Figure 4A; supplemental Table 4), and we also found that the levels of 2 metabolites involved in the ascorbate metabolism, diketogulonic acid and threonolactone, were increased in $\mathrm{HbS}$ cells (Figure 4B; supplemental Table 5).

In humans, conversion of arginine to ornithine and urea by arginase mainly occurs in liver, but arginase is also present in RBCs. ${ }^{27}$ Arginine could be imported from plasma to RBCs through the $\mathrm{y}+$ system, ${ }^{28}$ or might be produced in RBCs from aspartate as demonstrated for other cell type. ${ }^{29}$ Ornithine and urea may be products of arginine metabolism in RBCs. In HbS cells, decreased concentrations of ornithine and urea, as well as aspartate, were detected, whereas the concentration of fumarate, produced in the course of arginine biosynthesis from arginosuccinate, was increased (Figure 4B; supplemental Table 6). Outside the RBCs and in many cell types, ornithine is the precursor for polyamine synthesis. These polyamines (putrescine, spermine, and spermidine) can then penetrate and accumulate into the RBCs. Increased concentrations of spermidine and spermine were found in $\mathrm{RBCs}$ from SCD patients compared with controls, indicating an increased catabolism of ornithine (Figure 4B).

The nitric oxide (NO) pathway is a key element in SCD. ${ }^{30}$ Two metabolites, arginine (the NO synthase substrate) and citrulline (the NO synthase product), were detected in the RBC metabolome. No variation of arginine concentration was observed between SCD and normal RBCs, but the citrulline concentration was decreased in SCD RBCs. As RBCs do not have any NO synthase activity or urea cycle, citrulline might come from plasma (Figure 4A).

RBCs have an essential role in interorgan transport of amino acids resulting from many transport systems. ${ }^{31}$ In $\mathrm{HbS}$ cells, increased concentration of glycine, serine, glutamine, glutamate, and alanine, together with decreased concentrations of lysine and aspartate, were detected (Figure 4A; supplemental Tables 4, 6, and 7), whereas the levels of arginine, asparagine, methionine, phenylalanine, proline, threonine, tryptophan, tyrosine, and valine remained unchanged. Finally, carnitine and acetyl-carnitine concentrations were increased in $\mathrm{HbS}$ cells (Figure 4A; supplemental Table 7), and we observed an expected increased concentration of creatine (Figure 4B; supplemental Table 7), which accumulates in 
A

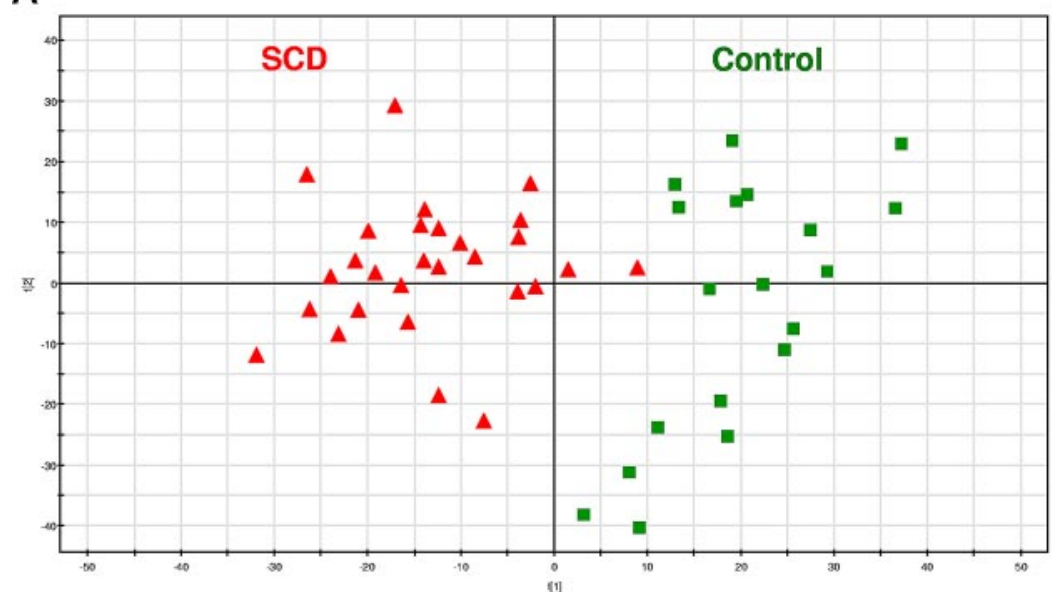

B

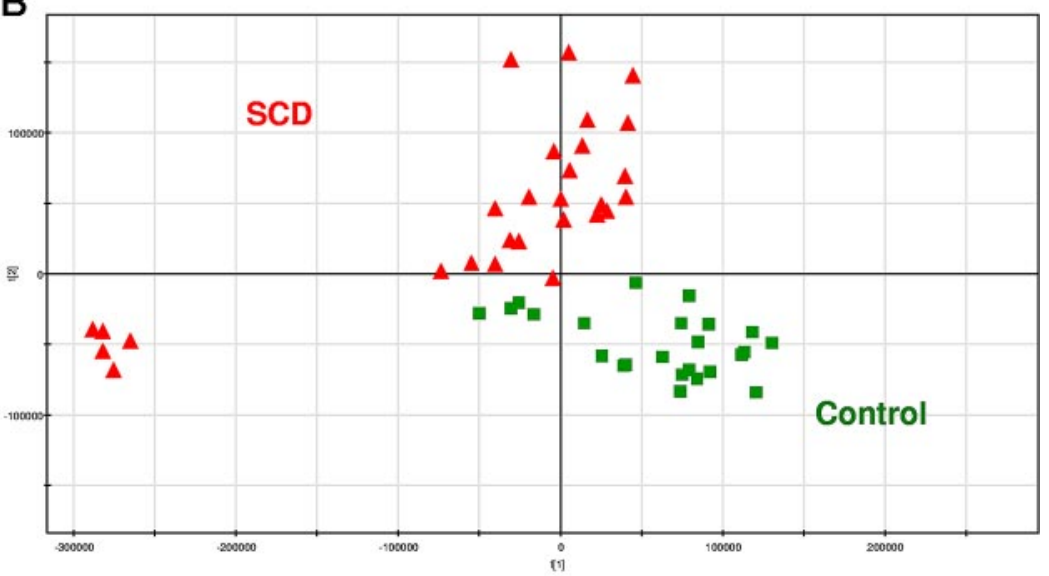

Figure 3. SCD patients and healthy subjects can be individualized on the basis of the metabolic information contained in their RBCs. PCA score plots for RBC extracts obtained from 28 SCD patients and 24 controls. Each point (red triangles and green squares for SCD patients and healthy subjects, respectively) represents an LC/MS metabolic fingerprint. (A) Data acquired in the negative mode of electrospray ionization. The variables contained in the resulting data matrix were meancentered and scaled to unit variance before PCA. The separation occurs on the first component. (B) Data acquired in the positive mode of ionization. The variables contained in the resulting data matrix were mean-centered and scaled to Pareto variance before PCA. The separation occurs on the second component. (C-D) Impact of the storage of RBC extracts on the metabolic information contained in the LC/MS fingerprints. Blood samples from SCD patients and controls (9 patients, and either 11 or 5 controls, for positive or negative ion mode, respectively) were collected and divided in 2 aliquots, one being immediately extracted and the other frozen, ${ }^{23}$ before future extraction. (C) PCA scores plot observed with detection in the positive ion mode. (D) Correlation between concentration ratios of SCD patients to controls obtained on fresh and frozen RBC extracts on 34 discriminating metabolites detected in positive ion mode.
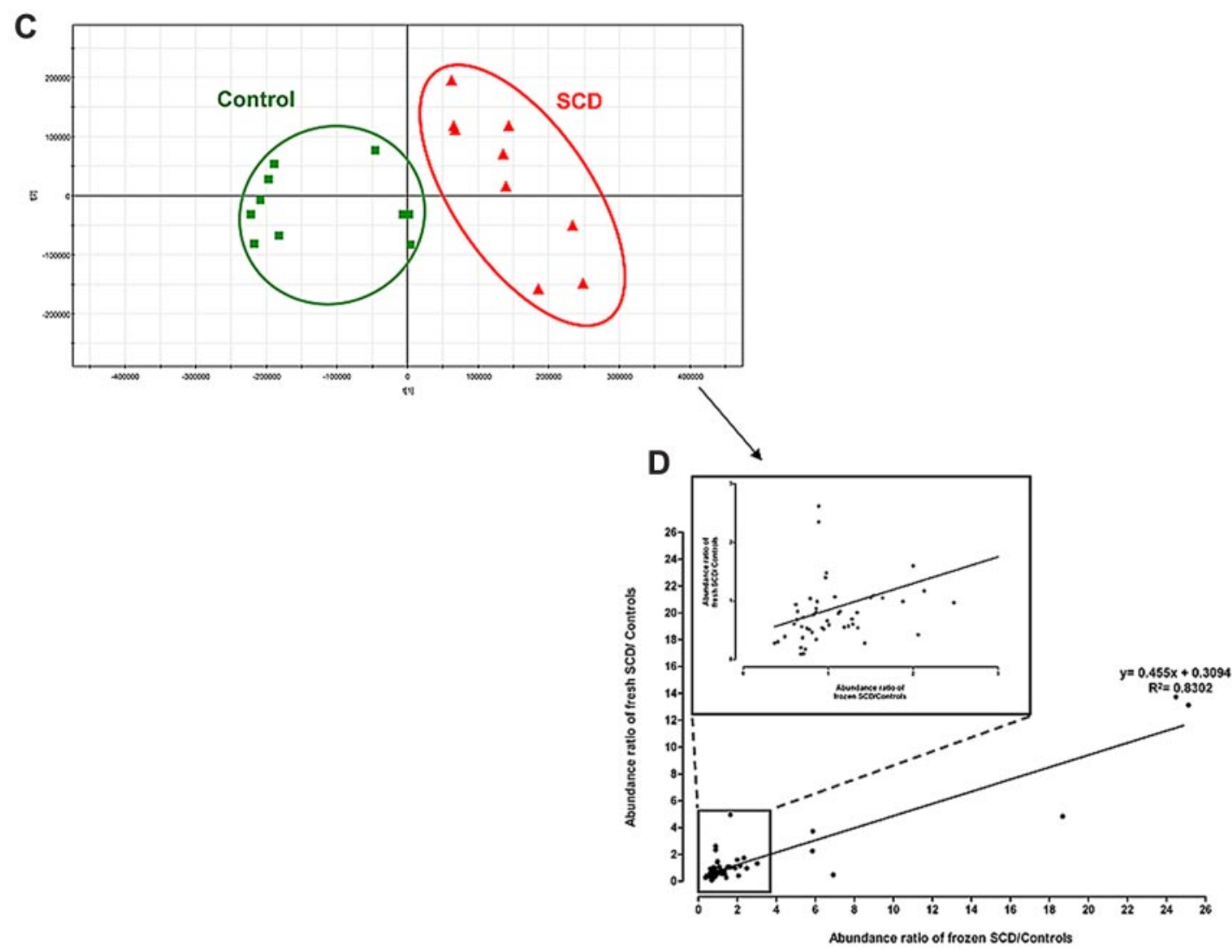

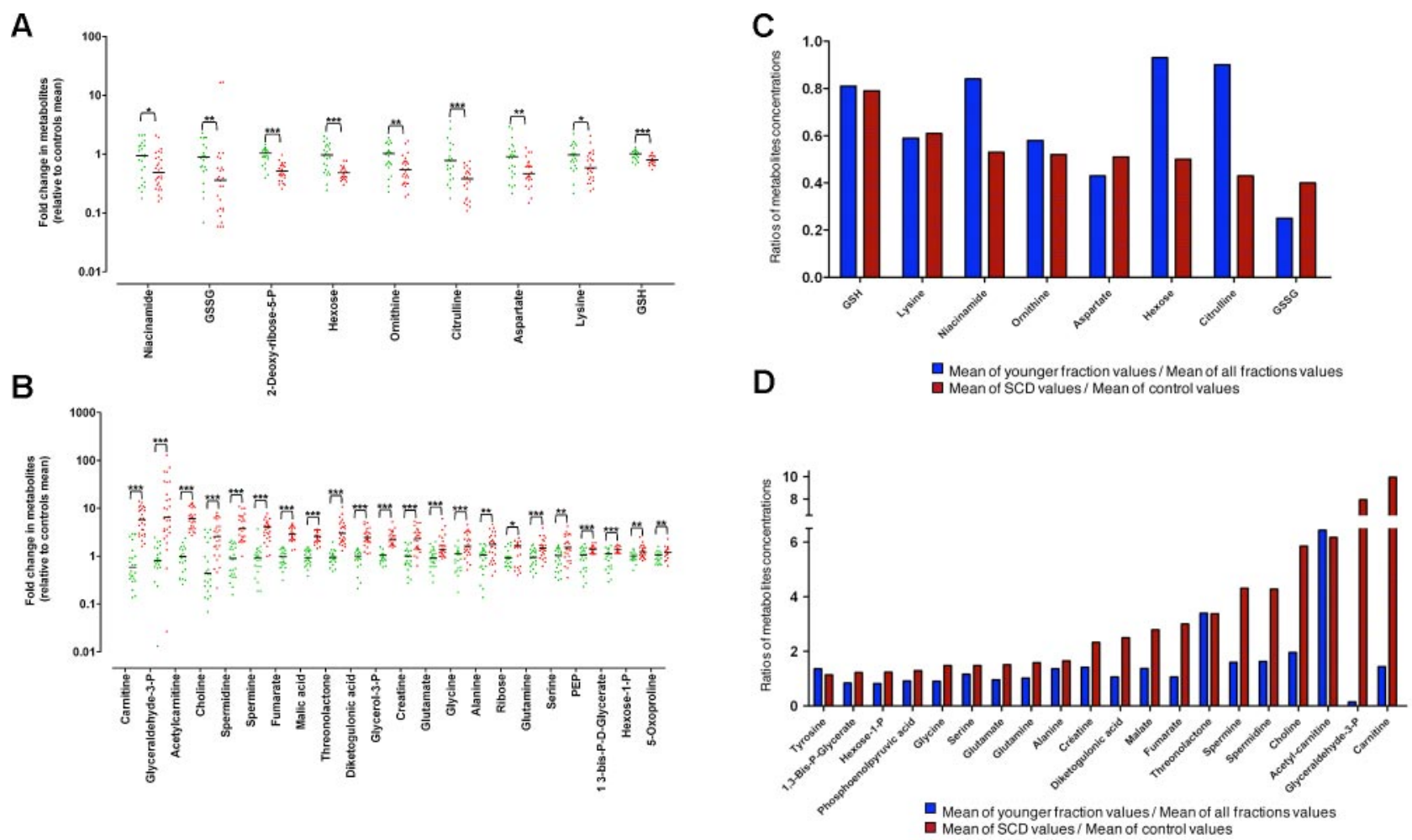

Figure 4. A metabolic signature of SCD patients and young RBCs can be obtained from LC/MS analysis of RBC extracts. Metabolite exhibiting significant concentration variations between controls and SCD patients. Values are ratios of individual levels observed for SCD patients (red points) and controls (green points) to mean levels obtained for healthy subjects. Statistically significant difference observed with a $t$ test: ${ }^{\star} .01<P<.05,{ }^{\star \star} .001<P<.01$, ${ }^{* \star} P<.001$. (A) Metabolites whose levels are decreased in SCD patients. (B) Metabolites whose levels are increased in SCD patients. (C) Metabolites whose levels are decreased in SCD patients were analyzed in young normal RBCs. (D) Metabolites whose levels are increased in SCD patients were analyzed in young normal RBCs. Values are ratios of mean levels observed for SCD patients and controls (red panel), compared with ratios of mean levels found for young RBCs and all aged RBCs (blue panel).

young $\mathrm{RBCs}^{32,33}$ and thus might reflect the short life span of the $\mathrm{HbS}$ cells.

To determine the impact of RBC age on the metabolic signature of SCD patients, we compared metabolomes of young RBCs from healthy subjects with those of RBCs from SCD patients. To this end, RBCs from controls were fractioned according to the age of the $\mathrm{RBCs}^{34}$ and metabolomes of the different fractions were analyzed by LC/MS. Then, we compared, for each discriminating metabolite previously identified, the variation of metabolite concentration between control and SCD RBCs, with the variation of metabolite concentration between young normal RBCs (ie, $<20$ days old) and normal RBCs. Any difference between these 2 ratios indicated that the variation of the metabolite concentration observed in SCD was not the result of the young age of the cell but to the pathology. By this mean, concentration trends of metabolites, such as citrulline, malate, or carnitine, were found to be specifically related to SCD, whereas those of metabolites, such as creatine, GSH, or serine, were found to be related to the youth of SCD RBCs (Figure 4C-D).

\section{Discussion}

Emerging metabolomics profiling technologies hold promise for illuminating biology and human diseases. We chose one of the less complex human cell subtypes, the RBC, and a very frequent and highly disabling disease, SCD, to identify biologically meaningful changes at the metabolite level. In this study, some compounds, such as glucose-6-phosphate and fructose-6-phosphate, or 1,3-
DPG and 2,3-DPG, cannot be separated using our LC/MS method. This limits biologic interpretations, unless the natural presence of a particular isomer in a given biologic medium or its level variations is supported by previously published data, as is the case with increased concentrations of 2,3-DPG in SCD disease. ${ }^{35,36}$ Finally, sample preparation (ie, RBC isolation and metabolite extraction) is a crucial step for metabolomics. Some described protocols rely on the use of thermal shocks ${ }^{37}$ or sonication ${ }^{38}$ for cell membrane disruption, followed by a metabolite extraction step using organic solvents. Compared with these methods, boiling RBCs at $100^{\circ} \mathrm{C}$ for 3 minutes in water followed by differential centrifugations gave the most reliable results in terms of number of metabolite detected and signal intensities. This protocol did not seem to hydrolyze hemoglobin, which represents $98 \%$ of cytoplasmic proteins, as the glutamic acid that is mutated to valine in sickle cell patients displayed a significantly higher level in $\mathrm{HbS}$ RBCs than in normal RBCs (Figure 4).

Almost all the life functions of RBCs are supported by energy from glycolysis. ${ }^{12}$ Although accumulations of glycolytic intermediates were observed in $\mathrm{HbS}$ cells, we detected neither any increased level of ADP or AMP nor any concentration variations of glycolysis end-products, such as pyruvate or lactate, thus indicating that glycolysis is not highly dysregulated in these cells. Interestingly, increased concentrations of malate were detected in $\mathrm{HbS}$ cells (Figure 5A). This organic acid can be produced from pyruvate with reduction of a $\mathrm{NAD}^{+}$molecule to $\mathrm{NADH}$, which might be involved in maintaining $\mathrm{HbS}$ cell redox balance. Indeed, reactive oxygen species production is increased in SCD, and a major consequence of reactive oxygen species production in $\mathrm{HbS}$ cells is the high 
A

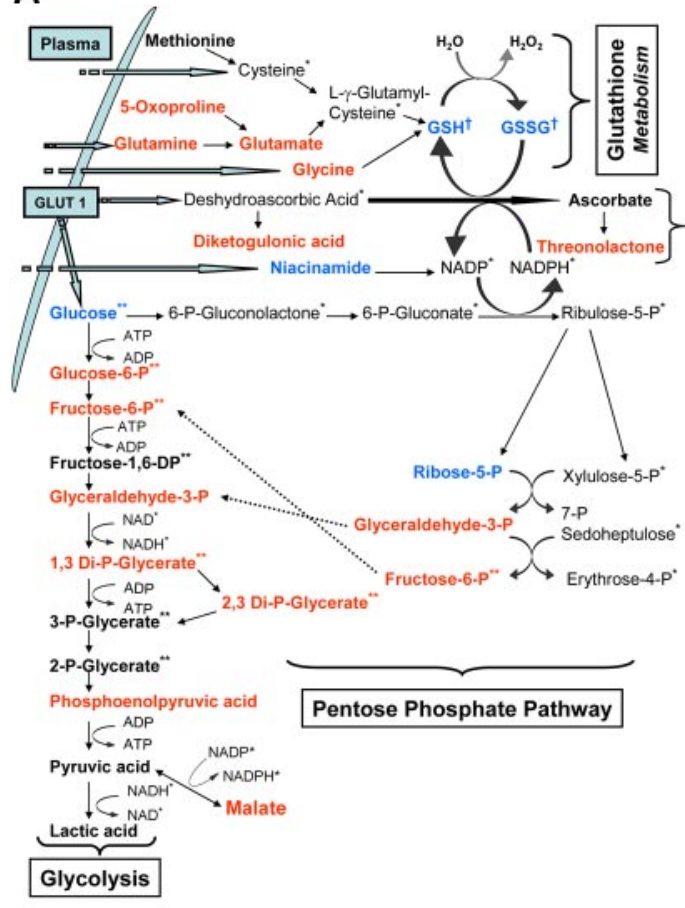

B

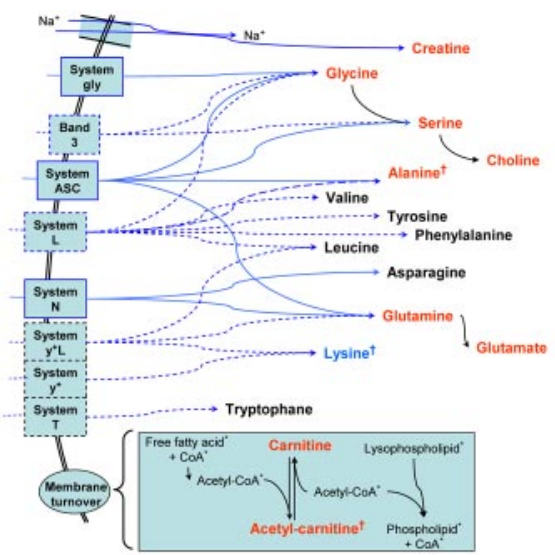

C

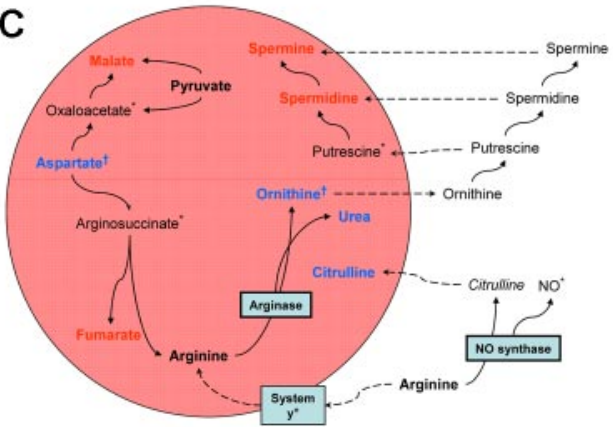

Figure 5. Alterations of specific pathways in $\mathrm{HbS}$ cells. Metabolites in red are increased in $\mathrm{HbS}$ cells versus normal RBCs. Metabolites in blue are decreased in $\mathrm{HbS}$ cells versus normal RBCs. Metabolites in black are unchanged in $\mathrm{HbS}$ cells versus normal RBCs. †Metabolites are related to RBC age and not SCD. *Metabolites are not detected in our study. ${ }^{\star \star}$ Metabolites cannot be set apart from its isomers. The solid black arrow indicates an enzymatic transformation; the solid blue arrow, a facilitated diffusion (passive transport) through RBC membrane; and the dashed blue arrow, a secondary active transport through the RBC membrane. (A) GSH metabolism, ascorbate metabolism, glycolytic activity, and pentose phosphate pathway. (B) Amino acid transport by RBC membrane and role of carnitine in cell membrane turnover. (C) Arginine and polyamine metabolism.

glutathione turnover. ${ }^{39,40}$ Accordingly, in $\mathrm{HbS}$ cells, decreased levels of GSH, GSSG, and Cys-Gly (nonsignificant: $P=.06$ ) and an increased concentration of GSH precursors, such as glutamine, glutamate, and glycine, were detected (Figure 5A). GSH and GSSG concentrations were also reduced in young $\mathrm{RBCs}$, suggesting that the variations observed in $\mathrm{HbS}$ cells may be related to the young age of RBCs in SCD. On the other hand, the high increase of glutamine, glutamate, and glycine in $\mathrm{HbS}$ cells did not seem to be accounted for by RBC age but directly related to SCD pathophysiology. The increased concentrations of these amino acids might probably be linked to increased plasma levels for glycine ${ }^{40}$ and/or to an increased activity of transport systems of glutamate and glutamine ${ }^{40}$ from the plasma ("system ASC" for glutamine and glycine transport, and "system gly" for glycine transport), whereas the catalytic activities of the enzymes involved in the glutathione biosynthesis pathway may also be saturated by such a substrate excess. Interestingly, the levels of other amino acids transported by the system ASC in RBCs (ie, alanine and serine; Figure 5B) are also increased in $\mathrm{HbS}$ cells, whereas serine is unchanged in the plasma of SCD patients. ${ }^{40}$

Otherwise, the pentose phosphate pathway catalyzes the reduction of NADP to NADPH and is important to ensure GSSG reduction. ${ }^{41}$ De novo synthesis of NADP requires the specific transport, involves niacinamide, and plays a significant role in GSSG reduction. Thus, decreased concentration of niacinamide in $\mathrm{HbS}$ cells might also be related to a high glutathione turnover.

RBCs can regenerate ascorbate ${ }^{42}$ from its oxidized form, dehydroascorbic acid (DHA). DHA is taken up from plasma by the glucose transport protein, GLUT1, and intracellular DHA is rapidly reduced to ascorbate by a GSH-dependent mechanism. ${ }^{43}$ Ascorbate is then slowly cleared from the cells but can also play an intracellular role for preventing lipid peroxidative damage in the erythrocyte membrane. ${ }^{44}$ The concentration of ascorbate was not significantly different between $\mathrm{HbS}$ cells and normal RBCs, but the concentration of diketogulonic acid, which is a direct degradation product of DHA, was increased (Figure 5A), suggesting that the capacity to reduce DHA might be overloaded because of a large import of DHA and/or because of a lack of GSH.

Oxidative stress from $\mathrm{HbS}$ auto-oxidation, increased binding of membrane-attack complex complement C $5 b-9,{ }^{45}$ shedding of membrane vesicles from repeated cycle of erythrocyte sickling and unsickling, and mechanical fragmentation under shear stress ${ }^{14,46}$ participated in $\mathrm{HbS}$ cell membrane fragility. Thus, membrane turnover and repair mechanisms are highly solicited in $\mathrm{HbS}$ cells, as emphasized by the observed increased concentrations of carnitine and acetyl-carnitine (Figure 5B), which are known to be used as buffer and reservoir of activated acyl group for the turnover and the repair of RBC membrane. ${ }^{47}$ Increased concentrations of choline (Figure 5B) were also detected in $\mathrm{HbS}$ cells and might be linked to recycling of phospholipids and thus document the RBC membrane fragility observed in SCD patients. Interestingly, the increased concentration of acetyl-carnitine might be related to the young age of HbS RBCs, whereas increased concentration of carnitine and choline might be directly related to SCD pathophysiology.

Endothelial dysfunctions are important actors of SCD vasculopathy, mostly because of impaired NO homeostasis. ${ }^{15}$ In SCD, intravascular hemolysis releases arginase from RBCs to blood plasma. Arginase competes with NO synthase, degrades arginine to ornithine, and severely limits NO synthesis (Figure 5C). This competition has a direct impact on clinical symptoms because 
sickle cell patients with the highest arginase activity in plasma and the lowest ratios of arginine to ornithine develop more vascular complications, such as pulmonary hypertension. ${ }^{48,49}$ We did not observe any altered arginine concentration in $\mathrm{HbS}$ cells, whereas citrulline, produced by NO synthase, was decreased. Ornithine concentration was decreased in both HbS RBCs and young RBCs, indicating that this metabolite level might be unrelated to SCD pathophysiology. Ornithine, which is synthesized by arginase, is an important precursor for proline and polyamines (putrescine, spermine, and spermidine) synthesis in many cell types. ${ }^{50}$ In HbS RBCs, the increased concentration of polyamines could not be accounted for by the young age of RBCs in SCD, and is probably linked to the pathology. The decreased citrulline concentration in $\mathrm{HbS}$ cells might reflect the competition between released arginase and NO synthase during intravascular hemolysis in sickle cell patients, and citrulline concentration could be a relevant biomarker of sickle cell vasculopathy that cannot currently be monitored by any biologic parameter.

In conclusion, a metabolic signature of SCD patients RBC that depicts the pathophysiology of SCD has been obtained from UHPLC/MS-based metabolome analysis of RBC extracts. Among the metabolites that participate in this signature, citrulline, spermine, and spermidine could be of interest for the follow-up of SCD patients and also for monitoring the effect of new drugs targeted against this pathology.

\section{Acknowledgments}

The authors thank Aurélie Roux, Wassim El Nemer, and Samia Boudah for helpful discussions and assistance in experiments and Dr Dora Bachir for help in collecting patient samples.
This work was supported by the Commissariat à l'Energie Atomique and Inserm. D.D., G.M., Y.X., and B.K. are supported by the Association pour la Recherche sur le Cancer, Inserm, Commissariat à l'Energie Atomique, and Académie de Médecine (fellowships). J.-F.H. is supported by the DIANE (Désordres Inflammatoires dans les Affections Neurologiques) project (Région wallonne, Belgium).

\section{Authorship}

Contribution: D.D. designed and performed research, analyzed data, performed statistical analysis, and wrote the manuscript; B.K. performed research, collected clinical data, analyzed data, and wrote the manuscript; G.M. performed the research and assisted with data analysis; J.-F.H. performed statistical analysis and critically reviewed manuscript; P. Bovee and G.B. performed the fractionation of RBCs according to their age; Y.X. performed research in MS and assisted with data analysis; M.-F.O. performed metabolite identification; P. Bartolucci, S.P., Y.C., and F.G. assisted with sample collection and provided patients' clinical data; M.B. collected clinical data; and P.-H.R. and C.J. supervised the project, designed research, and wrote the manuscript.

Conflict-of-interest disclosure: The authors declare no competing financial interests.

Correspondence: Paul-Henri Roméo, Commissariat à l'Energie Atomique/DSV/iRCM/LRTS, 92265 Fontenay-aux-Roses cedex, France; e-mail: paul-henri.romeo@cea.fr; or Christophe Junot, Commissariat à l'Energie Atomique/DSV/iBiTec-S/SPI, Bâtiment 136, Commissariat à l'Energie Atomique/Saclay, 91191 Gif-surYvette cedex, France; e-mail: christophe.junot@cea.fr.

\section{References}

1. Nicholson JK. Global systems biology, personalized medicine and molecular epidemiology. $\mathrm{Mol}$ Syst Biol. 2006;2:52.

2. Winder CL, Dunn WB, Schuler S, et al. Global metabolic profiling of Escherichia coli cultures: an evaluation of methods for quenching and extraction of intracellular metabolites. Anal Chem. 2008;80(8):2939-2948.

3. Lawton KA, Berger A, Mitchell M, et al. Analysis of the adult human plasma metabolome. Pharmacogenomics. 2008;9(4):383-397.

4. Kind T, Tolstikov V, Fiehn O, Weiss RH. A comprehensive urinary metabolomic approach for identifying kidney cancerr. Anal Biochem. 2007;363(2): 185-195.

5. Sreekumar A, Poisson LM, Rajendiran TM, et al. Metabolomic profiles delineate potential role for sarcosine in prostate cancer progression. Nature. 2009;457(7231):910-914.

6. Ritchie SA, Ahiahonu PW, Jayasinghe D, et al. Reduced levels of hydroxylated, polyunsaturated ultra long-chain fatty acids in the serum of colorectal cancer patients: implications for early screening and detection. BMC Med. 2010;8:13.

7. Oresic M, Simell S, Sysi-Aho M, et al. Dysregulation of lipid and amino acid metabolism precedes islet autoimmunity in children who later progress to type 1 diabetes. J Exp Med. 2008;205(13): 2975-2984

8. Lewis GD, Wei R, Liu E, et al. Metabolite profiling of blood from individuals undergoing planned myocardial infarction reveals early markers of myocardial injury. J Clin Invest. 2008;118(10): 3503-3512.

9. Sabatine MS, Liu E, Morrow DA, et al. Metabolomic identification of novel biomarkers of myocar- dial ischemia. Circulation. 2005;112(25):38683875.

10. Shaham O, Slate NG, Goldberger O, et al. A plasma signature of human mitochondrial disease revealed through metabolic profiling of spent media from cultured muscle cells. Proc Natl Acad Sci U S A. 2010;107(4):1571-1575.

11. Joshi A, Palsson BO. Metabolic dynamics in the human red cell: IV. Data prediction and some model computations. J Theor Biol. 1990;142(1): 69-85.

12. van WR, van Solinge WW. The energy-less red blood cell is lost: erythrocyte enzyme abnormalities of glycolysis. Blood. 2005;106(13):40344042.

13. Roux-Dalvai F, Gonzalez de PA, Simo C, et al. Extensive analysis of the cytoplasmic proteome of human erythrocytes using the peptide ligand library technology and advanced mass spectrom etry. Mol Cell Proteomics. 2008;7(11):2254-2269.

14. Stuart MJ, Nagel RL. Sickle-cell disease. Lancet. 2004;364(9442):1343-1360.

15. Wood KC, Hsu LL, Gladwin MT. Sickle cell disease vasculopathy: a state of nitric oxide resistance. Free Radic Biol Med. 2008;44(8):15061528.

16. Hankins J, Aygun B. Pharmacotherapy in sickle cell disease: state of the art and future prospects. Br J Haematol. 2009;145(3):296-308.

17. Nishino T, Yachie-Kinoshita A, Hirayama A, Soga T, Suematsu M, Tomita M. In silico modeling and metabolome analysis of long-stored erythrocytes to improve blood storage methods. J Biotechnol. 2009; 144(3):212-223

18. Darghouth D, Koehl B, Junot C, Romeo PH. Metabolomic analysis of normal and sickle cell erythrocytes. Transfus Clin Biol. 2010;17(3):148 150.

19. Smith CA, Want EJ, O'Maille G, Abagyan R, Siuzdak G. XCMS: processing mass spectrometry data for metabolite profiling using nonlinear peak alignment, matching, and identification. Anal Chem. 2006;78(3):779-787.

20. Trygg J, Holmes E, Lundstedt T. Chemometrics in metabonomics. J Proteome Res. 2007;6(2):469479 .

21. Tautenhahn R, Bottcher C, Neumann S. Highly sensitive feature detection for high resolution LC/MS. BMC Bioinformatics. 2008;9:504.

22. Croixmarie V, Umbdenstock $\mathrm{T}$, Cloarec $\mathrm{O}$, et al. Integrated comparison of drug-related and druginduced ultra performance liquid chromatography/mass spectrometry metabonomic profiles using human hepatocyte cultures. Anal Chem. 2009;81(15):6061-6069.

23. Mei H, Hsieh $\mathrm{Y}$, Nardo $\mathrm{C}$, et al. Investigation of matrix effects in bioanalytical high-performance liquid chromatography/tandem mass spectrometric assays: application to drug discovery. Rapid Commun Mass Spectrom. 2003;17(1):97-103.

24. Sumner LW, Amberg A, Barrett D, et al. Proposed minimum reporting standards for chemical analysis. Metabolomics. 2007;3(3):211-221.

25. Lelkens CC, Noorman F, Koning JG, et al. Stability after thawing of RBCs frozen with the highand low-glycerol method. Transfusion. 2003; 43(2):157-164.

26. Sasaki R, Ikura K, Narita H, Yanagawa S, Chiba H. 2,3-Bisphosphoglycerate in erythroid cells. Trends Biochem Sci. 1982;7(4):140-142.

27. Kim PS, lyer RK, Lu KV, et al. Expression of the 
liver form of arginase in erythrocytes. Mol Genet Metab. 2002;76(2):100-110.

28. Tunnicliff $G$. Amino acid transport by human erythrocyte membranes. Comp Biochem Physiol Comp Physiol. 1994;108(4):471-478.

29. Kanehisa M, Goto S. KEGG: kyoto encyclopedia of genes and genomes. Nucleic Acids Res. 2000; 28(1):27-30.

30. Reiter CD, Gladwin MT. An emerging role for nitric oxide in sickle cell disease vascular homeostasis and therapy. Curr Opin Hematol. 2003; 10(2):99-107.

31. Christensen HN. Interorgan amino acid nutrition. Physiol Rev. 1982;62(4):1193-1233.

32. Jiao $\mathrm{Y}$, Okumiya $\mathrm{T}$, Saibara $\mathrm{T}$, et al. An enzymatic assay for erythrocyte creatine as an index of the erythrocyte life time. Clin Biochem. 1998;31(2): 59-65.

33. Jiao YF, Okumiya T, Saibara T, Kudo Y, Sugiura T. Erythrocyte creatine as a marker of excessive erythrocyte destruction due to hypersplenism in patients with liver cirrhosis. Clin Biochem. 2001; 34(5):395-398.

34. Bosch FH, Werre JM, Roerdinkholder-Stoelwinder B Huls TH, Willekens FL, Halie MR. Characteristics of red blood cell populations fractionated with a combination of counterflow centrifugation and Percoll separation. Blood. 1992;79(1):254-260.

35. Poillon WN, Kim BC, Castro O. Intracellular hemoglobin S polymerization and the clinical severity of sickle cell anemia. Blood. 1998;91(5):17771783
36. Poillon WN, Kim BC, Labotka RJ, Hicks CU, Kark JA Antisickling effects of 2,3-diphosphoglycerate depletion. Blood. 1995;85(11):3289-3296.

37. Sana TR, Waddell K, Fischer SM. A sample extraction and chromatographic strategy for increasing LC/MS detection coverage of the erythrocyte metabolome. J Chromatogr B Analyt Technol Biomed Life Sci. 2008;871(2):314-321.

38. Xu F, Zou L, Lin Q, Ong CN. Use of liquid chromatography/tandem mass spectrometry and online databases for identification of phosphocholines and lysophosphatidylcholines in human red blood cells. Rapid Commun Mass Spectrom. 2009;23(19):3243-3254.

39. Reid M, Badaloo A, Forrester T, Jahoor F. In vivo rates of erythrocyte glutathione synthesis in adults with sickle cell disease. Am J Physiol Endocrinol Metab. 2006;291(1):E73-E79.

40. Kiessling K, Roberts N, Gibson JS, Ellory JC. A comparison in normal individuals and sickle cell patients of reduced glutathione precursors and their transport between plasma and red cells. $\mathrm{He}$ matol J. 2000;1(4):243-249.

41. Rabilloud T, Heller M, Gasnier F, et al. Proteomics analysis of cellular response to oxidative stress: evidence for in vivo overoxidation of peroxiredoxins at their active site. J Biol Chem. 2002;277(22): 19396-19401.

42. May JM. Ascorbate function and metabolism in the human erythrocyte. Front Biosci. 1998;3:d1d10.

43. May JM, Qu ZC, Whitesell RR, Cobb CE. Ascorbate recycling in human erythrocytes: role of
GSH in reducing dehydroascorbate. Free Radic Biol Med. 1996;20(4):543-551.

44. May JM. Is ascorbic acid an antioxidant for the plasma membrane? FASEB J. 1999;13(9):9951006.

45. Test ST, Woolworth VS. Defective regulation of complement by the sickle erythrocyte: evidence for a defect in control of membrane attack complex formation. Blood. 1994;83(3):842-852 .

46. Hagar W, Vichinsky E. Advances in clinical research in sickle cell disease. Br J Haematol. 2008;141(3):346-356.

47. Arduini A, Mancinelli G, Radatti GL, Dottori S, Molajoni F, Ramsay RR. Role of carnitine and carnitine palmitoyltransferase as integral components of the pathway for membrane phospholipid fatty acid turnover in intact human erythrocytes. J Biol Chem. 1992;267(18):12673-12681.

48. Kato GJ, Wang Z, Machado RF, Blackwelder WC, Taylor JG, Hazen SL. Endogenous nitric oxide synthase inhibitors in sickle cell disease: abnormal levels and correlations with pulmonary hypertension, desaturation, haemolysis, organ dysfunction and death. Br J Haematol. 2009;145(4): 506-513.

49. Morris CR, Kato GJ, Poljakovic M, et al. Dysregulated arginine metabolism, hemolysis-associated pulmonary hypertension, and mortality in sickle cell disease. JAMA. 2005;294(1):81-90.

50. Morris SM Jr. Regulation of enzymes of the urea cycle and arginine metabolism. Annu Rev Nutr. 2002;22:87-105. 